\title{
Robotic-assisted parathyroidectomy via transaxillary approach: feasibility and learning curves
}

\author{
Emad Kandil $^{1}$, Deena Hadedeya ${ }^{1}$, Mahmoud Shalaby ${ }^{1}$, Eman Toraih $^{1,2}$, David Aparício ${ }^{3}$, Meghan Garstka $^{1}$, \\ Ruhul Munshi ${ }^{1}$, Ahmed Elnahla ${ }^{1}$, Jonathon O. Russell ${ }^{4}$, Patrick Aidan ${ }^{3}$ \\ ${ }^{1}$ Department of Surgery, Tulane University School of Medicine, New Orleans, LA, USA; ${ }^{2}$ Genetic Unit, Department of Histology and Cell Biology, \\ Faculty of Medicine, Suez Canal University, Ismailia, Egypt; ${ }^{3}$ Department of Otorhinolaryngology and Head and Neck Surgery, American Hospital of \\ Paris, Paris, France; ${ }^{4}$ Department of Otolaryngology-Head and Neck Surgery, Johns Hopkins University School of Medicine, Baltimore, MD, USA \\ Contributions: (I) Conception and design: D Hadedeya, M Shalaby, E Toraih, E Kandil; (II) Administrative support: E Kandil, P Aidan; (III) \\ Provision of study materials or patients: D Hadedeya, M Shalaby, E Kandil, P Aidan; (IV) Collection and assembly of data: D Hadedeya, M Shalaby, \\ D Aparício, R Munshi, A Elnahla; (V) Data analysis and interpretation: E Toraih; (VI) Manuscript writing: All authors; (VII) Final approval of \\ manuscript: All authors. \\ Correspondence to: Emad Kandil, MD, MBA, FACS. Department of Surgery, Tulane University School of Medicine, 1430 Tulane Ave., SL-22, New \\ Orleans, LA 70112, USA. Email: ekandil@tulane.edu.
}

Background There have been few reports of robotic-assisted transaxillary parathyroidectomy in the literature. We aim to report our experience with robotic-assisted transaxillary parathyroidectomy for primary hyperparathyroidism (PHPT) in the Western population.

Methods: A retrospective study was performed from July 2010 through July 2019 at two institutions, one in the United States and one in France. Demographic characteristics and perioperative data were collected for all patients undergoing robotic-assisted transaxillary parathyroidectomy by a single surgeon at each institution. A linear regression model was developed to describe the learning curve for this procedure at each institution.

Results: One-hundred and two patients with PHPT were included with a median age of 55.6 \pm 12.4 years and median body mass index (BMI) of $25.5 \pm 6.1 \mathrm{~kg} / \mathrm{m}^{2}$. The majority of patients were female $(80.4 \%)$. Median total operative time was $116 \pm 53$ minutes. Minor complications were reported in 2 patients $(1.96 \%)$, and one case was converted to a trans-cervical approach (TCA) for four-gland exploration. Median patient follow-up time was $6.5 \pm 12.2$ months, and disease recurrence was reported in one patient. Calculated learning curves showed that one surgeon achieved proficiency by the eighth case, and the other achieved proficiency by the fourteenth case.

Conclusions: This is the largest reported experience of robotic-assisted transaxillary parathyroidectomy for PHPT in the Asian and Western population. Analysis of the procedural learning curve demonstrates that proficiency in this technique was achieved after performance of less than 15 surgeries. This procedure is safe and feasible in the hands of experienced surgeons for select patients with localized disease.

Keywords: Hyperparathyroidism; parathyroid adenoma; parathyroidectomy; robotic parathyroidectomy; transaxillary parathyroidectomy

Submitted Oct 15, 2020. Accepted for publication Feb 05, 2021.

doi: $10.21037 /$ gs-20-761

View this article at: http://dx.doi.org/10.21037/gs-20-761 


\section{Introduction}

Surgical treatment for primary hyperparathyroidism (PHPT) involved the standard of bilateral cervical exploration and localization for all four glands with removal of grossly enlarged glands until the 1990s (1). Subsequent improvements in the accuracy and reliability of preoperative localization studies have allowed for the development of targeted minimally invasive surgical approaches to the parathyroid glands. Targeted parathyroidectomy with intraoperative parathyroid hormone (IOPTH) currently constitutes the co-gold-standard procedure for PHPT with results equivalent to bilateral cervical exploration and has become the preferred procedure by most endocrine surgeons $(2,3)$. Various approaches to avoid a neck scar via minimally invasive or remote incisional access have been developed for parathyroidectomy (2). In particular, the robotic-assisted transaxillary approach to the thyroid and subsequently parathyroid glands was developed in South Korea and adopted by surgeons in the United States and Europe who have published various case reports and studies on small series of these procedures as they require an experienced surgeon with high volume of cases $(2,4)$. In addition to that, the long procedure time, and the high cost when it compared to the conventional approach make them less popular (4).

As the authors have also continued to perform a large series of robotic-assisted parathyroid surgeries, we seek in this study to present and analyze the data from our cumulative experience, so as to evaluate the safety and feasibility of robotic-assisted transaxillary parathyroidectomy in patients with PHPT in a series of procedures performed in the Western population at our respective North American and European institutions. We present the following article in accordance with the STROBE reporting checklist (available at http://dx.doi.org/10.21037/gs-20-761).

\section{Methods}

This is a two institutions study of retrospectively collected data from two tertiary centers, Tulane Medical Center in New Orleans, Louisiana, USA and the American Hospital of Paris in Paris, France. This study was approved by the institutional review boards of Tulane Medical Center (IRB reference number: 2020-264). Individual consent for this retrospective study was waived. The study was conducted in accordance with the Declaration of Helsinki (as revised in 2013). Patients who underwent robotic-assisted transaxillary parathyroidectomy from July 2010 to July 2019 for management of PHPT were included. Demographic characteristics and perioperative data including the complications and outcomes were collected, analyzed, and reported. The surgical technique was performed as previously described by the authors in earlier publications (3,5-7). Both surgeons have a relatively the same level of robotic experience in neck surgeries. The operation was not offered to patients with previous neck irradiation, suspicion of malignancy (parathyroid carcinoma), previous neck surgery, nonlocalized/equivocal disease, suspicion of multi-gland disease, neck mobility problems and cervical spine disease, or with significant comorbidities, as defined by having an American Society of Anesthesiologists Classification greater than two (ASA >2). All patients underwent ultrasound and sestamibi scans for preoperative localization. Flexible laryngoscopy was performed in all patients preoperatively and postoperatively. These cases represent the initial cases of transaxillary parathyroidectomy by both surgeons. Cure was defined based on Miami criteria as a $>50 \%$ PTH drop from either the greatest pre-incision or pre-excision PTH measurement in a sample of blood drawn 10 min following complete resection of a hyperfunctioning gland.

For the purposes of defining a learning curve, operative time was used as a surrogate for procedural proficiency. Operative time was defined as the time of incision to the time of skin closure and was plotted as a function of case number. A simple moving average (SMA) of order 3 was then calculated for the series, in a method similar to prior literature describing the learning curves for remote-access approaches to the thyroid (8).

\section{Statistical analysis}

A linear regression model was used to evaluate the learning curve of first 25 and 56 cases in both centers, respectively, that involved robotic-assisted transaxillary parathyroidectomy for a single adenoma without concomitant thyroidectomy procedures. This analysis was performed to define time for proficiency. The proficiency point was defined as the point at which the slope significantly changed, dividing the curve into two phases: the skill acquisition phase (prior to the proficiency point), and the proficiency phase (after the proficiency point).

\section{Results}

One-hundred and two patients with confirmed PHPT underwent robotic-assisted transaxillary parathyroidectomy. 


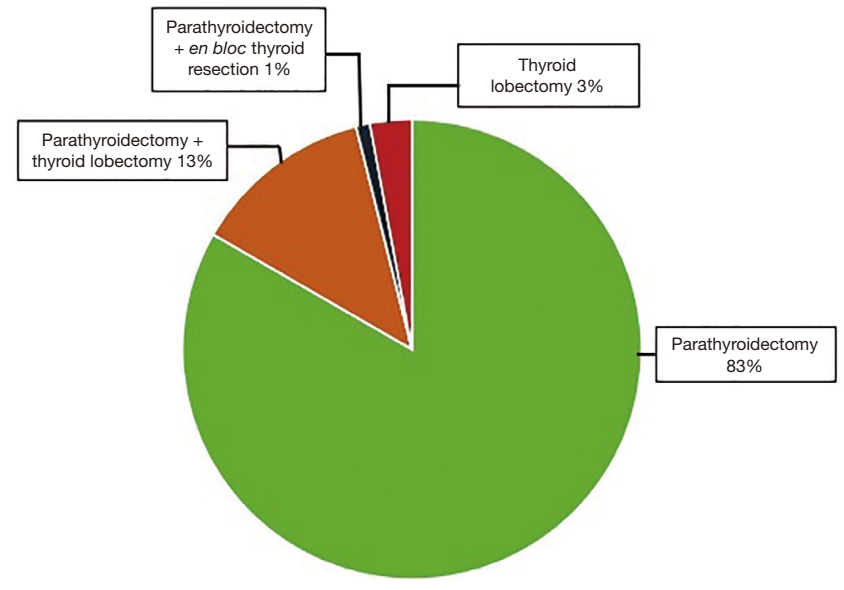

Figure 1 Type of surgery.

Eighty-two of these patients (80.4\%) were females. The median age of the patient population was $55.6 \pm 12.4$ years, and the median body mass index (BMI) was $25.5 \pm 6.1 \mathrm{~kg} / \mathrm{m}^{2}$. The median preoperative serum PTH level was $151.9 \pm 95.6 \mathrm{pg} / \mathrm{mL}$ and the median preoperative serum calcium level was $11.1 \pm 0.9 \mathrm{mg} / \mathrm{dL}$. Eighty-five patients underwent parathyroidectomy for parathyroid adenomas ( 2 of them were intrathymic), 3 patients underwent a thyroid lobectomy for intrathyroidal parathyroid adenoma that was confirmed preoperatively with FNA and PTH washout, 1 patient underwent parathyroidectomy with an en bloc resection of the right thyroid lobe for an atypical parathyroid adenoma (suspicious for malignancy), and 13 patients underwent thyroid lobectomy concurrent with parathyroidectomy for a thyroid lesion, Figure 1. For those who underwent parathyroidectomy only, the median total operative time was $116 \pm 53$ minutes. With regards to robotic surgical parameters, the median docking time was $5.9 \pm 2.6$ minutes, and the median console time was $38.9 \pm 23.5$ minutes. The median intraoperative blood loss was $20 \pm 11 \mathrm{~mL}$.

Intraoperative monitoring of serum intact PTH levels was performed, and in all but one case there was a greater than $50 \%$ drop in serum PTH level value after excision of the prelocalized parathyroid glands. In one patient, the serum PTH level remained high after excision of the targeted gland, and the operation was converted to a conventional open trans-cervical approach (TCA) for bilateral neck exploration and subtotal parathyroidectomy. After the subtotal parathyroidectomy, IOPTH dropped more than $50 \%$. The median weight of the resected parathyroid glands was $1.1 \pm 0.8 \mathrm{gm}$. Seventy-three patients $(71.5 \%)$ were discharged on the day of the surgical procedure, and the remaining 29 patients (28.5\%) were observed overnight and discharged on the first post-operative day. Post-operative laboratory values demonstrated a median 1-week postoperative serum PTH level of $39 \pm 22.1 \mathrm{pg} / \mathrm{mL}$ and median postoperative serum calcium level of $9.2 \pm 0.64 \mathrm{mg} / \mathrm{dL}$. Median duration of follow-up was $6.5 \pm 12.2$ months for this cohort, and recurrent PHPT was reported in one case, Table 1.

Two patients $(1.96 \%)$ developed postoperative complications. One seroma, defined as collection of clear serous fluid under the skin at the incision site with no signs of infection including pus formation, occurred and was managed conservatively, resolving after 10 days. One superficial wound infection was managed with oral antibiotics in outpatient setting. Flexible laryngoscopy was performed at the first postoperative visit and confirmed normal vocal cord mobility in all patients, and there were no reported cases of permanent vocal cord paralysis.

A linear regression model was used to evaluate the learning curve for the first 25 and 56 robotic-assisted transaxillary single-adenoma parathyroidectomy cases in both centers, respectively. At Tulane Medical Center, proficiency was obtained after the $8^{\text {th }}$ case with a decline in operative time from 170 to 100 minutes. Subsequent to this drop, there was a stabilization in mean operative time at $95.6 \pm 13.5$ minutes. At the American Hospital of Paris, proficiency was obtained at the $14^{\text {th }}$ case, with a decline from over 300 to 126 minutes. The latter part of the curve showed continuous gradual improvement, reaching a median operative time of $91.5 \pm 33.9$ minutes. With continuous improvement, both surgeons were able to finish the operation in 40 minutes, Figures 2,3. There was no significant difference in patients' BMI between the two institutions ( $\mathrm{P}$ value $>0.05$ ). Also, there was no difference in the patients' BMI during the learning phase and the proficiency phase, Figure 4.

\section{Discussion}

Remote-access techniques, including robotic-assisted transaxillary surgery, have proven to be effective and safe for thyroid surgery in patients with specific selection and when performed by high-volume surgeons (9-15). The literature on remote access techniques for parathyroidectomy has consisted mostly of small case series (4,9,16-19). This includes the results of initial experience of the authors with the robotic-assisted transaxillary gasless approach to parathyroidectomy at our North American institution in 2014 , in which we documented a series of nine patients 
Table 1 Characteristics of patients with PHPT undergoing robotic-assisted transaxillary parathyroidectomy

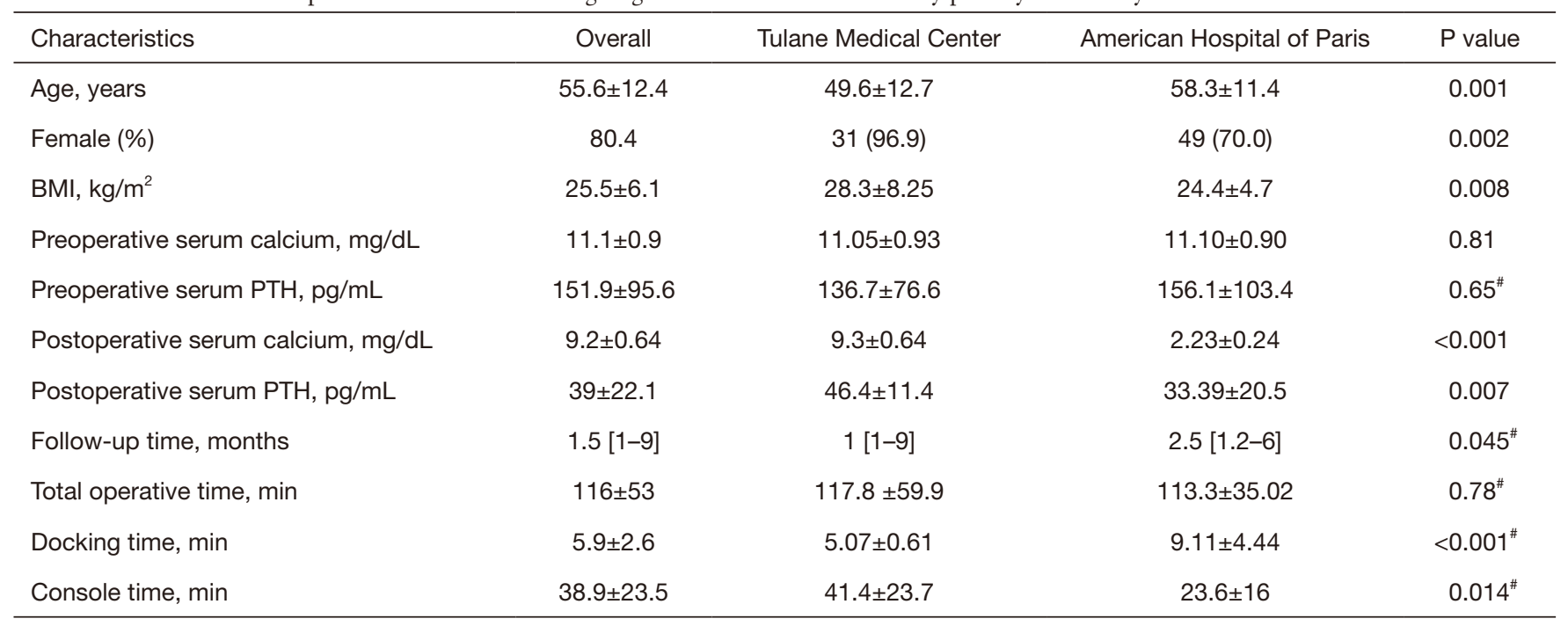

Data presented as numbers (percentage), median [quartile], or mean and standard deviation. Two-sided Chi square was used for categorical variables and independent student-t or Mann Whitney $U\left({ }^{\#}\right)$ test were applied for quantitative variables. PHPT, primary hyperparathyroidism; BMI, body mass index; PTH, parathyroid hormone.

with confirmed PHPT who underwent surgery between September 2010 and March 2011 (18). Similarly, the first experience of the authors with the robotic-assisted transaxillary and retroauricular approaches to thyroid and parathyroid surgery in the European population was documented in a small series by Boccara et al., who reported on the anesthetic implications of these procedures with a series of twenty cases in 2013, including 14 patients undergoing thyroid lobectomy, 4 undergoing total thyroidectomy, and 2 undergoing parathyroidectomy (7). The current work represents the combined experience of these authors with robotic-assisted transaxillary parathyroidectomy in the Western population over the past decade, analyzing a series of 102 patients undergoing parathyroidectomy. To our knowledge, this represents one of the largest reported series of robotic-assisted transaxillary parathyroidectomy in the literature.

The robotic-assisted transaxillary approach has many advantages which have been documented previously in the literature for thyroid surgery. It does not require carbon dioxide $\left(\mathrm{CO}_{2}\right)$ insufflation $(20,21)$. The use of the surgical robot allows three-dimensional (3D) visualization which gives more perceptional depth and magnification, in addition to an increased amount of free motion in the robotic arms, which allows for precise tissue manipulation and dissection within a limited working space, such as the neck or superior mediastinum (16,21-23) This access to the superior mediastinum has allowed for robotic removal of ectopic glands, as documented by Ismail et al. (24). The current series documented by the authors included two patients with intrathymic adenomas removed successfully by the robotic-assisted transaxillary technique.

Limitations of robotic-assisted remote access approaches for neck surgery documented in the literature include the need for an experienced, high-volume surgeon, strict patient selection criteria, prolonged operative time compared to open surgeries, and the high cost of the procedure $(20,21)$. One of the unique limitations to the robotic-assisted remote access approaches is the complications related to the flap as a larger area of subcutaneous tissue dissection is required with this approach. Studies shows contradictory results as some reported a less pain with the robotic-assisted remote access approach and other reported a mild post-operative symptom related to the flap including pain and paresthesia (21). The mean operative time for this series was $116 \pm 53$ minutes, which is shorter than others documented previously in the literature, likely due to a larger volume of cases in the series $(4,16)$. Our learning curve and proficiency analysis demonstrated a progressive reduction in total operative time from over 5 hours to under an hour. We also note that various factors can impact the total operative time, including familiarity of the entire operating room team with the procedure, since teams who perform a large volume of these cases will be used to the equipment and processes involved. 
A

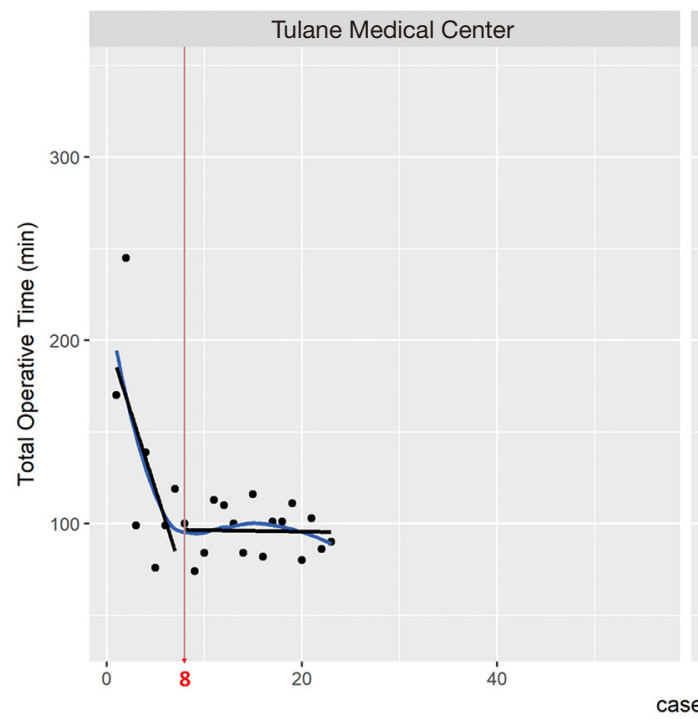

B



Figure 2 Learning curve to assess the rate of progression and mastery of the robotic transaxillary parathyroidectomy. X-axis represents the number of cases arranged by order, while the $\mathrm{Y}$-axis represents the time taken during the procedure. As the surgeon learns to operate the robot following the procedural steps, he becomes faster and more proficient at using the machine. Dispersion around the line highlights the variations and outliers. The slopes of learning phase $(-16.714 x+202.14$ and $-13.514 x+274.09$, respectively) were compared with the slopes of proficiency phase $(-0.0779 x+97.146$ and $-1.1039 x+131.15$, respectively $)$, and they were significantly different $(\mathrm{P}<0.001)$.

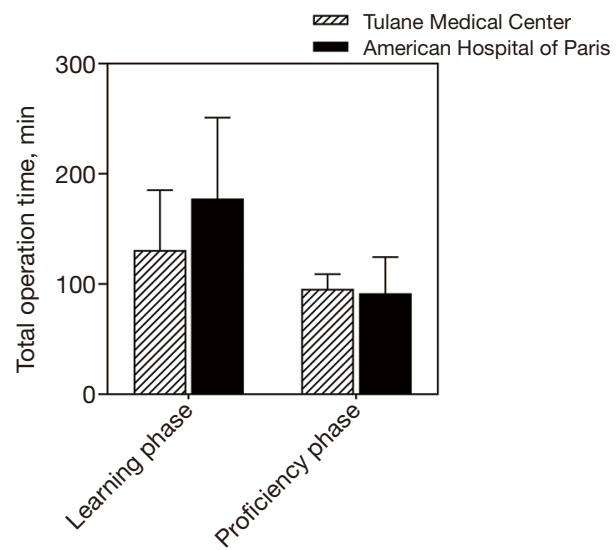

Figure 3 Mean total operative time during the learning phase and the proficiency phases. Student's $t$-test was used. Tulane Medical Center: learning phase (130.8 554.5 minutes) and proficiency phase (95.6 613.5 minutes). The American Hospital of Paris:

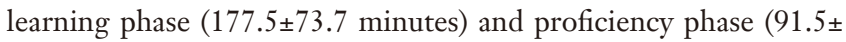
33.2 minutes)

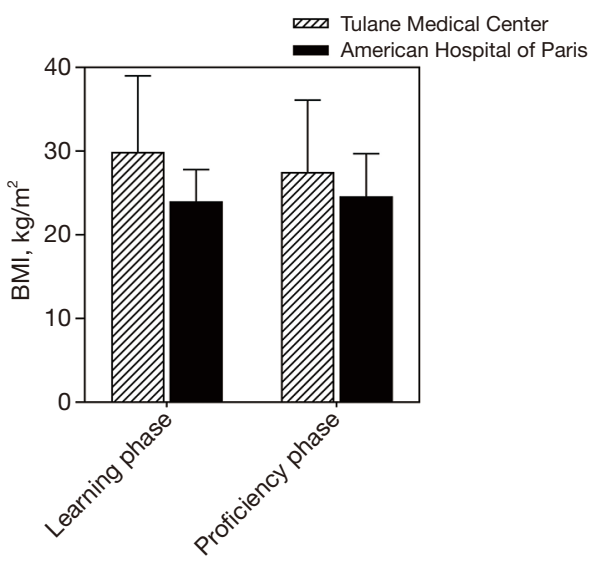

Figure 4 Comparison between the two centers according to $\mathrm{BMI}$ in $\mathrm{kg} / \mathrm{m}^{2}$ during the learning phase and the proficiency phase. Tulane Medical Center: mean BMI in the learning phase is $\left(29.9 \pm 9.1 \mathrm{~kg} / \mathrm{m}^{2}\right)$ and in the proficiency phase is $\left(27.5 \pm 8.6 \mathrm{~kg} / \mathrm{m}^{2}\right)$. The American Hospital of Paris: mean BMI in the Learning phase is $\left(24.0 \pm 3.8 \mathrm{~kg} / \mathrm{m}^{2}\right)$ and in the Proficiency phase is $\left(24.6 \pm 5.1 \mathrm{~kg} / \mathrm{m}^{2}\right)$. BMI, body mass index. 
Despite these reported limitations, robotic-assisted transaxillary parathyroidectomy is a viable option for patients who are motivated to avoid a visible neck scar. Various studies in the literature have addressed this topic, with some studies noting that patient interest in having a scarless neck procedure was as high as $65 \%$ to $75 \%$ of those surveyed $(25,26)$. Eye-tracking technology has shown that neck scars are noticeable enough to draw the visual attention from the face to the scar site $(27,28)$. Additionally, studies of the Dermatology Life Quality Index (DLQI), a questionnaire used to measure the impact of skin diseases and scars on patients' quality of life, have demonstrated that DLQI values are much higher in patients with post-thyroidectomy neck scars (9.02) than those with hypertrophic and keloid scars (7.79, normal control 0.58) (29). The mean DLQI value in patients with postthyroidectomy neck scars was also slightly higher than the DLQI of patients with psoriasis (8.73) (30). These findings highlight the potential benefits of remote-access surgical techniques for thyroid and parathyroid surgery such as the remote-access transaxillary parathyroidectomy highlighted in our study.

Potential complications of robotic-assisted transaxillary parathyroid surgery include brachial plexus injury from patient positioning, as well as damage to structures including the internal jugular vein, trachea, esophagus, and thoracic duct. None of these major complications had been reported in the literature, but the risk is still present $(11,17,21,31)$. In this study, the complication rate from robotic-assisted transaxillary parathyroidectomy is generally comparable with that reported from the conventional cervical approach (32). We routinely perform monitoring somatosensory evoked potentials (SSEP) during robotic-assisted transaxillary surgery (Biotronic, Ann Arbor, MI, USA) to avoid brachial plexus injury, as described previously by the authors (33). However, many other robotic surgeons do not use SSEP monitoring and have been able to avoid this serious complication by careful positioning of the arm. With regards to disease-specific complications, our data showed evidence of recurrence in one case, with a mean post-surgical follow-up time 6.5 months. None of our patients developed persistent disease. The risk of treatment failure (recurrence or persistent disease) is similar to that documented for the conventional cervical parathyroidectomy approach $(34,35)$.

\section{Conclusions}

Robotic-assisted transaxillary parathyroidectomy is a safe and feasible procedure for select patients with PHPT when conducted by experienced surgeons. Proficiency was achieved in both cases in fewer than 15 cases. Patients who are motivated to avoid a cervical incision should be considered for remote access parathyroidectomy options including robotic-assisted transaxillary surgery.

\section{Acknowledgments}

Funding: None.

\section{Footnote}

Reporting Checklist: The authors have completed the STROBE reporting checklist. Available at http://dx.doi. org/10.21037/gs-20-761

Data Sharing Statement: Available at http://dx.doi. org/10.21037/gs-20-761

Conflicts of Interest: All authors have completed the ICMJE uniform disclosure form (available at http://dx.doi. org/10.21037/gs-20-761). EK serves as the Editor-in-Chief of Gland Surgery. The other authors have no conflicts of interest to declare.

Ethical Statement: The authors are accountable for all aspects of the work in ensuring that questions related to the accuracy or integrity of any part of the work are appropriately investigated and resolved. The study was approved by the institutional review board of Tulane Medical Center (IRB 2020-264) and individual consent for this retrospective analysis was waived. The study was conducted in accordance with the Declaration of Helsinki (as revised in 2013).

Open Access Statement: This is an Open Access article distributed in accordance with the Creative Commons Attribution-NonCommercial-NoDerivs 4.0 International License (CC BY-NC-ND 4.0), which permits the noncommercial replication and distribution of the article with the strict proviso that no changes or edits are made and the original work is properly cited (including links to both the formal publication through the relevant DOI and the license). See: https://creativecommons.org/licenses/by-nc-nd/4.0/.

\section{References}

1. Noureldine SI, Gooi Z, Tufano RP. Minimally invasive 
parathyroid surgery. Gland Surg 2015;4:410-9.

2. Garas G, Holsinger FC, Grant DG, et al. Is robotic parathyroidectomy a feasible and safe alternative to targeted open parathyroidectomy for the treatment of primary hyperparathyroidism? Int J Surg 2015 Mar; 15:55-60.

3. Mohamed HE, Bhatia P, Aslam R, et al. Robotic transaxillary and retroauricular parathyroid surgery. Gland Surg 2015;4:420-8.

4. Landry CS, Grubbs EG, Morris GS, et al. Robot assisted transaxillary surgery (RATS) for the removal of thyroid and parathyroid glands. Surgery 2011;149:549-55.

5. Mohsin K, Alzahrani H, Bu Ali D, et al. Robotic transaxillary parathyroidectomy. Gland Surg 2017;6:410-1.

6. Kandil EH, Noureldine SI, Yao L, et al. Robotic transaxillary thyroidectomy: an examination of the first one hundred cases. J Am Coll Surg 2012;214:558-64; discussion 564-6.

7. Boccara G, Guenoun T, Aidan P. Anesthetic implications for robot-assisted transaxillary thyroid and parathyroid surgery: a report of twenty cases. J Clin Anesth 2013;25:508-12.

8. Razavi CR, Vasiliou E, Tufano, RP, et al. Learning curve for transoral endoscopic thyroid lobectomy. Otolaryngol Head Neck Surg 2018;159:625-9.

9. Russell JO, Clark J, Noureldine SI, Anuwong A, et al. Transoral thyroidectomy and parathyroidectomy - A North American series of robotic and endoscopic transoral approaches to the central neck. Oral Oncol 2017;71:75-80.

10. Russell JO, Razavi CR, Garstka ME, et al. Remote-access thyroidectomy: a multi-institutional north american experience with transaxillary, robotic facelift, and transoral endoscopic vestibular approaches. J Am Coll Surg 2019;228:516-22.

11. Ban EJ, Yoo JY, Kim WW, et al. Surgical complications after robotic thyroidectomy for thyroid carcinoma: a single center experience with 3,000 patients. Surg Endosc 2014;28:2555-63.

12. Berber E, Bernet V, Fahey TJ 3rd, et al. American Thyroid Association statement on remote-access thyroid surgery. Thyroid 2016;26:331-7.

13. Kandil E, Hammad AY, Walvekar RR, et al. Robotic thyroidectomy versus nonrobotic approaches: a metaanalysis examining surgical outcomes. Surg Innov 2016;23:317-25.

14. Choi JY, Lee KE, Chung KW, et al. Endoscopic thyroidectomy via bilateral axillo-breast approach (BABA): review of 512 cases in a single institute. Surg Endosc
2012;26:948-55.

15. Arora A, Garas G, Sharma S, et al. Comparing transaxillary robotic thyroidectomy with conventional surgery in a UK population: a case control study. Int J Surg 2016;27:110-7.

16. Karagkounis G, Uzun DD, Mason DP, et al. Robotic surgery for primary hyperparathyroidism. Surg Endosc 2014;28:2702-7.

17. Sasanakietkul T, Jitpratoom P, Anuwong A. Transoral endoscopic parathyroidectomy vestibular approach: a novel scarless parathyroid surgery. Surg Endosc 2017;31:3755-63.

18. Noureldine SI, Lewing N, Tufano RP, et al. The role of the robotic-assisted transaxillary gasless approach for the removal of parathyroid adenomas. ORL J Otorhinolaryngol Relat Spec 2014;76:19-24.

19. Ozdenkaya Y, Ersavas C, Arslan NC. Robotic transoral vestibular parathyroidectomy: two case reports and review of literature. World J Clin Cases 2018;6:542-7.

20. Brunaud L, Li Z, Van Den Heede K, et al. Endoscopic and robotic parathyroidectomy in patients with primary hyperparathyroidism. Gland Surg 2016;5:352-60.

21. Arora A, Garas G, Tolley N. Robotic parathyroid surgery: current perspectives and future considerations. ORL J Otorhinolaryngol Relat Spec 2018;80:195-203.

22. Tolley N, Arora A, Palazzo F, et al. Robotic-assisted parathyroidectomy: a feasibility study. Otolaryngol Head Neck Surg 2011;144:859-66.

23. Witzel K, von Rahden BH, Kaminski C, et al. Transoral access for endoscopic thyroid resection. Surg Endosc 2008;22:1871-5.

24. Ismail M, Maza S, Swierzy M, et al. Resection of ectopic mediastinal parathyroid glands with the da Vinci robotic system. Br J Surg 2010;97:337-43.

25. Arora A, Swords C, Garas G, et al. The perception of scar cosmesis following thyroid and parathyroid surgery: a prospective cohort study. Int J Surg 2016;25:38-43.

26. Wong W, Foo FJ, Lau MI, et al. Simplified minimally invasive parathyroidectomy: a series of 100 cases and review of the literature. Ann R Coll Surg Engl 2011;93:290-3.

27. Liao D, Ishii LE, Chen LW, et al. Transoral neck surgery prevents attentional bias towards the neck compared to open neck surgery. Laryngoscope 2020;130:1603-8.

28. Juarez MC, Ishii L, Nellis JC, et al. Objectively measuring social attention of thyroid neck scars and transoral surgery using eye tracking. Laryngoscope 2019;129:2789-94.

29. Balci DD, Inandi T, Dogramaci CA, et al. DLQI scores in patients with keloids and hypertrophic scars: a prospective 
case control study. J Dtsch Dermatol Ges 2009;7:688-92.

30. Choi Y, Lee JH, Kim YH, et al. Impact of postthyroidectomy scar on the quality of life of thyroid cancer patients. Ann Dermatol 2014;26:693-9.

31. Karakas E, Steinfeldt T, Gockel A, et al. Transoral thyroid and parathyroid surgery--development of a new transoral technique. Surgery 2011;150:108-15.

32. Khan MA, Rafiq S, Lanitis S, et al. Surgical treatment of primary hyperparathyroidism: description of techniques and advances in the field. Indian J Surg 2014;76:308-15.

Cite this article as: Kandil E, Hadedeya D, Shalaby M, Toraih E, Aparício D, Garstka M, Munshi R, Elnahla A, Russell JO, Aidan P. Robotic-assisted parathyroidectomy via transaxillary approach: feasibility and learning curves. Gland Surg 2021;10(3):953-960. doi: 10.21037/gs-20-761
33. Huang S, Garstka M, Murcy M, et al. Somatosensory evoked potential: preventing brachial plexus injury in transaxillary robotic surgery. Laryngoscope 2019;129:2663-8.

34. Hedbäck G, Odén A. Recurrence of hyperparathyroidism; a long-term follow-up after surgery for primary hyperparathyroidism. Eur J Endocrinol 2003;148:413-21.

35. Lou I, Balentine C, Clarkson S, et al. How long should we follow patients after apparently curative parathyroidectomy? Surgery 2017;161:54-61. 